Un sujet de grande importance

\section{en physique est la réponse}

d'un matériau soumis à un

rayonnement, que ce soit la

couleur d'un objet dans la

lumière du soleil, les dégâts

$d^{\prime}$ irradiation ou I'utilisation

de la spectroscopie pour

sonder la matière. La tâche

d'un(e) théoricien(ne) consiste

à structurer ce problème

complexe, et à trouver des

approximations qui permettent

de franchir le grand pas

séparant les modèles simples

des matériaux réels.

Cet article donne un aperçu

des défis actuels, du langage

que nous utilisons pour aborder

le problème, et des applications

possibles.

\title{
Théorie des excitations électroniques : une histoire complexe
}

Lucia Reining (lucia.reining@polytechnique.fr)

Laboratoire des solides irradiés, UMR 7642, École polytechnique, 91128 Palaiseau Cedex

Comprendre l'interaction entre rayonnement et matériau est un des grands défis auquel est confrontée la théorie de la matière condensée : il s'agit en effet de décrire, comprendre et prévoir comment un système collectif de l'ordre de $10^{23}$ électrons réagit à une perturbation. Or, les électrons ne sont pas indépendants, mais interagissent fortement entre eux via l'interaction coulombienne : on dit qu'ils forment un système corrélé. Il existe plusieurs méthodes pour aborder ce problème ; on en rencontre ainsi en chimie ou en physique, dénommées par des sigles tels que CI (Configuration Interaction), QMC (Quantum Monte Carlo), MBPT (Many-Body Perturbation Theory), DMFT (Dynamical Mean Field Theory), et bien d'autres [1]. Il serait intéressant de comprendre et comparer toute cette "zoologie " - mais ici, nous essayerons juste de nous focaliser sur l'essence du problème. En effet, la première question qui se pose est tout simplement : qu'est-ce qu'un « effet de corrélation "?

\section{Interactions, corrélations et observations}

Trop souvent, le mot « corrélation » est utilisé pour dénommer tout ce que l'on ne comprend pas. On peut se faire une idée du problème en considérant les excitations électroniques. Dans un matériau dans lequel les particules sont supposées indépendantes, chaque électron occupe un niveau d'énergie. Une excitation (par exemple, l'absorption d'un photon) se traduit par le passage d'un électron de son niveau initial (où il laisse un trou) à un niveau vide dans le matériau, voire en dehors si l'énergie transmise est assez grande. Les autres électrons restent spectateurs (fig. 1a). Mais dans la réalité, chaque excitation affecte l'ensemble des électrons. En revenant à l'ensemble des particules représenté sur la figure 1, on peut s'imaginer que l'on excite un électron, mais que le système entier réagit. Par exemple, comme illustré par la figure $1 b$,
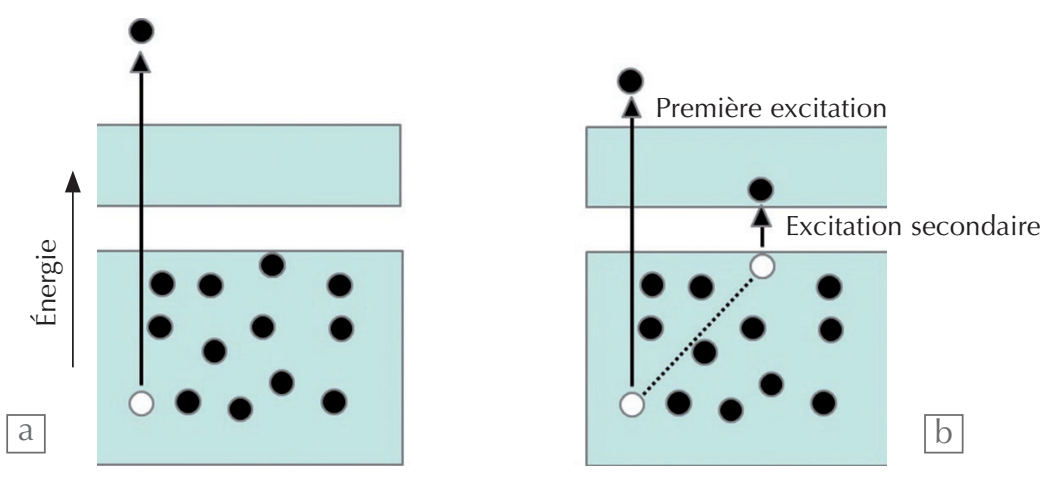

1. Illustration schématique d'une excitation dans un système d'électrons indépendants (a) et dans un système d'électrons en interaction (b). Les rectangles verts représentent l'ensemble des états occupés (en bas) et inoccupés (en haut), séparés par une bande interdite, dans l'état initial d'un matériau semi-conducteur. Les électrons et les trous sont indiqués respectivement par des cercles noirs et blancs. 
La ligne de lumière CASSIOPÉE du synchrotron SOLEIL, dédiée à la spectroscopie à haute résolution des électrons photoémis par les solides excités par des rayons $X$ mous.

le premier électron excité peut aussi céder de l'énergie à un autre couple électron-trou via l'interaction coulombienne (indiquée par un trait en pointillés). Ainsi, la première excitation a une durée de vie finie et d'autres excitations apparaissent.

On mesure les excitations électroniques en faisant de la spectroscopie, par exemple dans une expérience de photoémission où un photon de haute énergie éjecte un électron du matériau (voir encadré 1). On peut aussi injecter un électron et mesurer l'énergie des photons émis : c'est la photoémission inverse, qui donne des informations sur les niveaux électroniques inoccupés situés au-dessus du niveau de Fermi.

La figure 2 montre schématiquement le spectre, ou "fonction spectrale ", que l'on obtient dans une expérience de photoémission. Sans interaction, on trouverait simplement une raie étroite (correspondant à l'énergie de l'excitation dans la figure 1a).

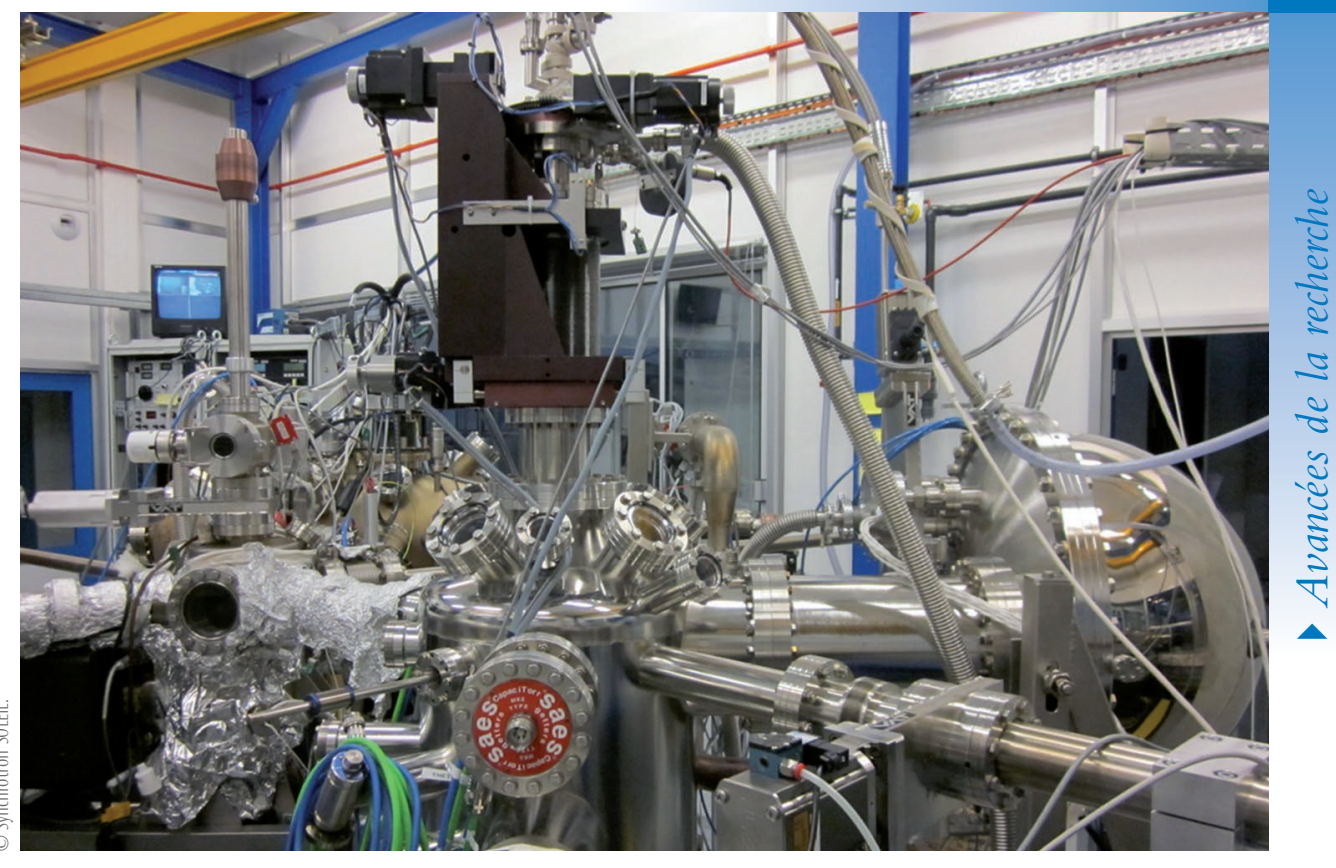

Les interactions entraînent un déplacement en énergie, car le système peut se relaxer pour minimiser son énergie. De plus, l'excitation a une durée de vie $\tau$ finie. $\mathrm{La}$ relation de Heisenberg $\tau \Delta \mathrm{E}=\hbar$ entre durée de vie et incertitude en énergie $\Delta \mathrm{E}$ prévoit alors un élargissement de la raie. On obtient ce qu'on appelle un pic de " quasi-particule ", pour souligner le changement par rapport au point de départ, la raie étroite correspondant à l'excitation d'une seule particule. On voit aussi un « satellite " à énergie plus basse. Les satellites sont dus aux excitations secondaires comme, par exemple, celle montrée dans la figure $1 b$ : comme une partie de l'énergie reste dans le matériau sous forme d'excitations, l'électron émis sort avec une énergie plus faible. Finalement, on trouve un continuum d'excitations possibles, donnant lieu en général à une fonction spectrale assez complexe, qui montre bien l'effet de la corrélation!

\〉

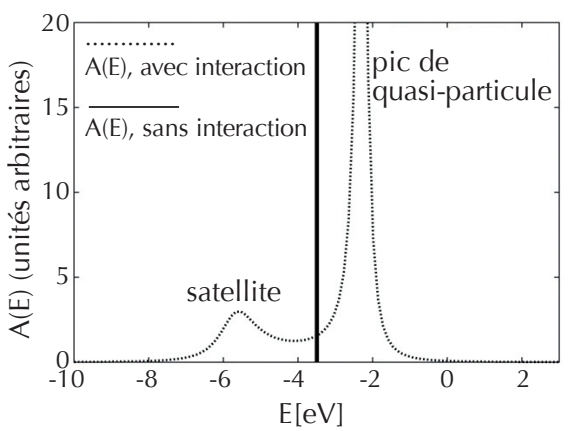
de liaison dans le cas typique d'un solide.

Ligne verticale : excitation d'une particule dans un système sans interaction. Courbe en pointillés : fonction spectrale d'un système en interaction. On voit le pic de "quasiparticule » vers $-2 \mathrm{eV}$ et un « satellite » vers $-6 \mathrm{eV}$. Leur distance, quelques eV, est de l'ordre de grandeur de l'énergie d'une excitation collective (" plasmon ») dans un métal simple (voir par exemple la figure 5a).
2. Fonctions spectrales $A(E)$ en fonction de l'énergie

\section{La photoémission}

encadré 1

La photoémission, ou spectroscopie de photoélectrons (fig. E1), est fondée sur l'effet photoélectrique : elle consiste à irradier un échantillon avec un faisceau lumineux monochromatique d'énergie suffisante pour arracher des électrons, typiquement des rayons $X$ ou de la lumière ultraviolette. On mesure l'énergie cinétique des électrons émis. La différence entre l'énergie des photons et cette énergie cinétique nous renseigne sur la variation d'énergie de l'échantillon, entre avant la mesure et après avoir perdu l'électron.

Dans un schéma avec des particules indépendantes (fig. 1a), cette différence d'énergie correspond simplement au niveau d'énergie que l'électron arraché occupait dans le matériau. Le résultat de la mesure (nombre de photoélectrons en fonction de leur énergie) sera alors une «fonction spectrale » $A(E)$, formée d'un seul pic ("fonction $\delta$ ») à l'énergie en question, indiqué par le trait vertical dans la figure 2 . Si on tient compte des interactions, la fonction spectrale devient la distribution en énergie des excitations de l'échantillon ayant perdu un électron (courbe en pointillés de la figure 2), conformément au dessin de la figure $1 \mathrm{~b}$. L'abscisse de la fonction spectrale est typiquement l'énergie initiale des électrons émis par rapport à celle d'un électron au niveau de Fermi. Cette énergie, qui est donc négative, s'appelle «énergie de liaison ».

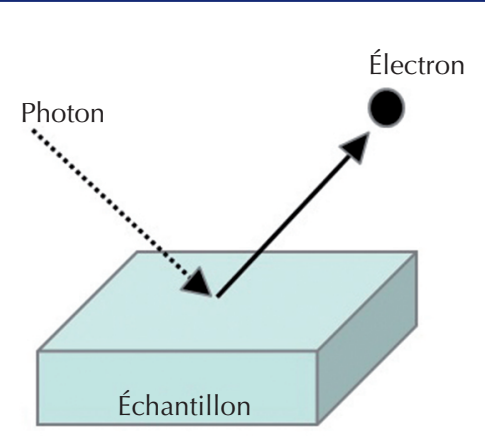

E1. Schéma de principe d'une expérience de photoémission. 
La physique d'un système à plusieurs électrons peut se décrire en termes de processus de propagation, de diffusion, d'échange et de polarisation. Les équations qui décrivent ces processus et leurs possibles combinaisons sont complexes. Pour avoir une représentation plus compacte et pour faciliter l'interprétation, on peut utiliser un langage graphique, les diagrammes. Cet encadré en présente les briques de base (fig. E2), qui sont utilisées dans les équations représentées dans la figure 3.

La fonction de Green à une particule représente la propagation dans le temps d'une particule d'un endroit à un autre. Nous représentons ceci par un vecteur. Pour un électron isolé, $c^{\prime}$ est le premier diagramme de la figure E2, le vecteur fin (a). Pour un électron en interaction avec d'autres électrons, nous utilisons un vecteur gras (b). L'interaction coulombienne elle-même est représentée par une ligne pointillée (c). Par contre, dans un matériau, l'interaction effective est écrantée ; on utilise alors la ligne ondulée (d). Cet écrantage est l'effet d'une polarisation, autrement dit, de la création de paires électron-trou : ce sont deux fonctions de Green qui forment une paire (une pour l'électron, l'autre pour le trou); il s'agit d'une boucle, car on représente la propagation du trou dans le sens opposé à la propagation d'un électron (e). Finalement, les deux derniers éléments sont deux processus de base : le premier montre l'interaction coulombienne avec la densité de charge, qui apparaît dans le potentiel classique de Hartree $(f)$. Le deuxième est le terme d'échange, propre aux fermions, dû au fait qu'on ne peut pas les distinguer (g).

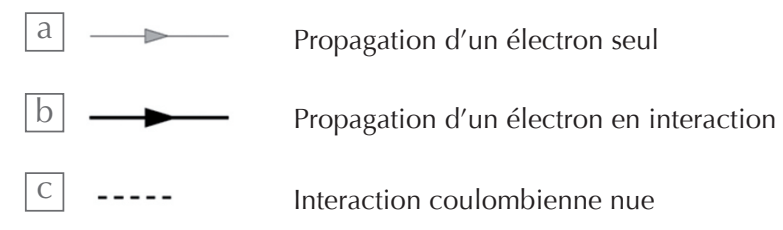

d Inn Interaction coulombienne ecrantée

$\mathrm{e} \bigcirc$ Paire électron-trou

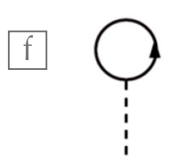

Interaction coulombienne avec la densité de charge

Terme d'échange ou de Fock

E2. Les principaux éléments des diagrammes de Feynman.

\section{\〉}

L'interaction a donc des effets qualitatifs, bien au-delà et bien plus intéressants qu'un simple déplacement en énergie du spectre. Les conséquences de ces effets d'interaction peuvent être très importantes, influant sur des phénomènes tels que les transitions de phase ou la supraconductivité.

\section{Comprendre les mécanismes : une question de langage}

Est-il possible de calculer et de prévoir ces effets ? En principe, nous savons ce qu'il faudrait faire : résoudre l'équation de Schrödinger «multi-particules». Cependant, avec $10^{23}$ électrons, ce n'est pas réaliste [2]. Le travail d'un(e) théoricien(ne) dans notre domaine consiste donc à réécrire cette équation en trouvant des approximations astucieuses qui contiennent la physique qui nous intéresse, tout en rendant possible le calcul de la solution - le plus souvent par ordinateur.

Pour trouver une bonne formulation, il faut tout d'abord mettre en place un langage. Celui que nous avons adopté entre dans le cadre des "fonctions de Green ». Ces fonctions sont les briques de base de ce que nous voulons décrire : l'histoire des électrons qui se déplacent dans le matériau. Une «fonction de Green à une particule » est l'objet mathématique qui décrit la propagation d'une particule dans le système en présence de toutes les autres (il y a aussi une fonction de Green à deux particules qui décrit la propagation de deux électrons, deux trous ou d'une paire électron-trou, et ainsi de suite). Bien sûr, ce sont des objets plutôt compliqués sur le plan mathématique, peu adaptés à une première approche du sujet ! Cependant, il suffit parfois de changer de langage pour mieux se faire comprendre... et pour notre problème, on peut trouver une formulation compacte et intuitive avec un langage "diagrammatique " introduit par Feynman. Il s'agit d'une approche graphique pour représenter les formules bien trop longues [3]. L'encadré 2 présente les différents éléments de ce langage.

Ces éléments sont alors combinés pour écrire l'histoire de la propagation d'un électron, comme le montre la figure $3 \mathrm{a}$. On voit que cette propagation (le vecteur gras) est différente de celle d'un électron seul (vecteur fin), car il y a l'interaction coulombienne (ligne pointillée) classique de notre particule avec la densité de charge de tous les électrons, c'est-à-dire la " contribution de Hartree ». De plus, les électrons étant des fermions, on ne peut pas les distinguer. Pour cette raison, on peut échanger des bouts de fonctions de Green, ce qui donne le diagramme suivant (dit " terme de Fock»).

Est-ce tout ? Bien sûr que non. Imaginons la particule qui se propage dans le système comme un bateau sur l'eau (fig. 4a). L'eau porte et freine le bateau (effet similaire au terme de Hartree), mais elle n'est pas inerte : le bateau crée des ondes, qui elles-mêmes vont avoir un effet important sur le bateau (il suffit de penser au mal de mer...).

De même, la particule qui se propage influence le système. Si on s'imagine en première approche la densité électronique comme une matière continue et déformable, comme l'eau, alors on comprend que des ondes se forment, par exemple quand on introduit un trou ou un électron dans le système (fig. 4b). Chaque particule transporte alors avec elle une déformation de la densité de charge - la " quasi-particule » est cet ensemble. C'est cette quasi-particule qui crée le pic principal en pointillés dans la figure 2. L'interaction effective entre 


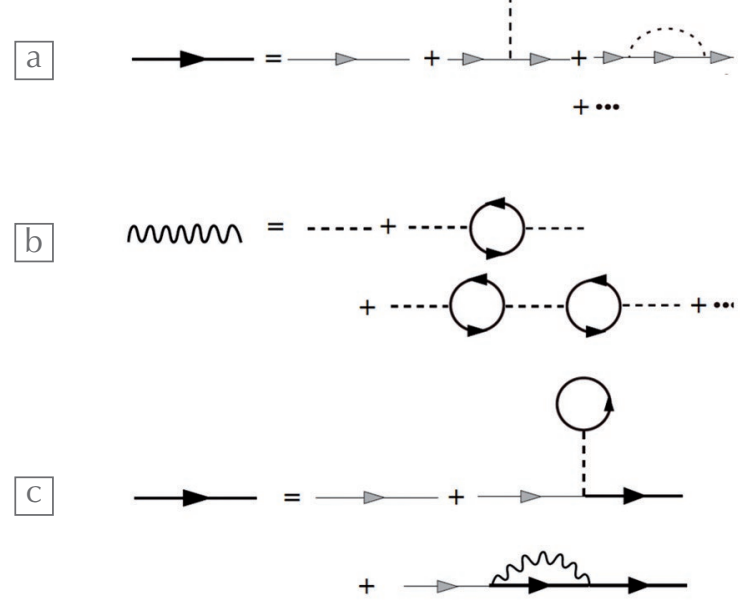

3. Les processus qui ont lieu pendant la propagation d'un électron dans un matériau peuvent être représentés dans le langage des diagrammes.

(a) La première ligne montre les premières contributions (dites « Hartree » et « Fock ») à la propagation d'une particule. La deuxième ligne (b) montre l'écrantage de l'interaction coulombienne par la création de paires électron-trou (approximation appelée « RPA »). La troisième ligne (c) montre une équation de Dyson dans une approximation appelée " GW », avec le terme de Hartree et le terme de Fock écranté.
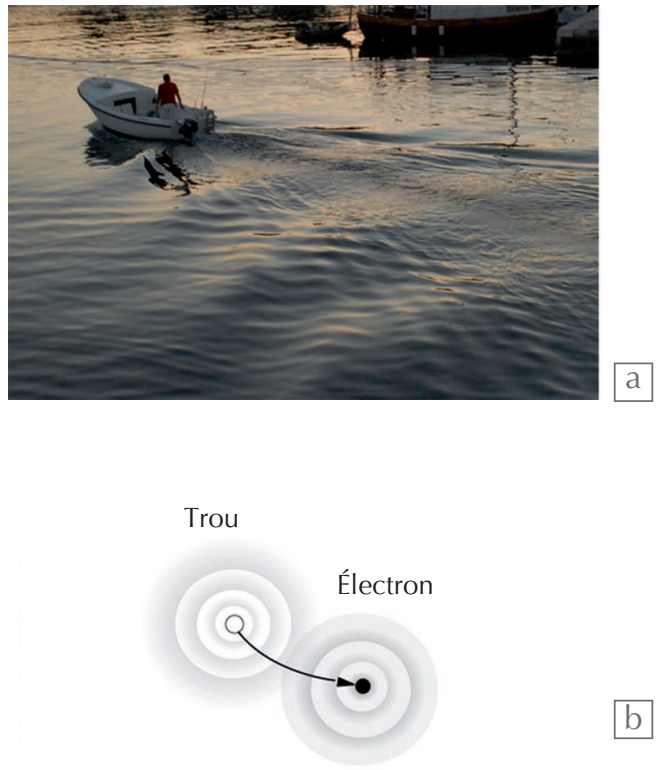

4. Perturbation d'un milieu par un objet qui s'y propage.

(a) Une particule qui se propage dans un système perturbe ce dernier, comme un bateau crée des ondes dans l'eau (permission L. Sponza).

(b) Dans un système d'électrons, ce sont des ondes de densité de charge qui apparaissent quand on crée une paire électron (cercle noir)-trou (cercle blanc). quasi-particules est différente, et généralement plus faible, que l'interaction coulombienne entre particules isolées car elle est écrantée. Cet écrantage est dynamique, comme les ondes. On appelle les ondes de densité de charge des "plasmons".

Dans notre langage diagrammatique, si l'interaction coulombienne nue est représentée par les pointillés, l'interaction effective, écrantée, est décrite par une ligne ondulée. Quel est le lien entre les deux ? L'effet de l'interaction est de déplacer les particules. Il y a donc, par rapport au système initial, un endroit où une particule vient de disparaître (on parle d'un trou), et un autre endroit où une particule apparait. On dit qu'on crée des paires électron-trou. La modification de l'interaction qui en résulte est montrée dans les diagrammes de la figure $3 \mathrm{~b}$. Il s'agit d'une description simplifiée, appelée " approximation de la phase aléatoire " ou "RPA ». On néglige le fait que chaque électron et chaque trou dans les paires ainsi créées interagissent à leur tour. Les formules correspondant aux diagrammes RPA peuvent être évaluées numériquement et donnent souvent des résultats assez satisfaisants.

\section{Des mots aux résultats}

Les ondes de densité de charge, ou plasmons, peuvent être créées par la propagation d'un électron du système, mais aussi par une perturbation externe telle que des rayons $\mathrm{X}$ ou un faisceau d'électrons dans un microscope électronique. C'est un peu comme la perturbation d'un ensemble de personnes dans une salle : un mouvement collectif peut être causé par une personne qui commence à courir, ou bien par une influence extérieure, comme le dessert qui arrive... Si on utilise une perturbation externe, on peut directement mesurer la réponse collective. Par exemple, un faisceau d'électrons ou de rayons $\mathrm{X}$ interagit avec l'échantillon et est réfléchi ou transmis. Il perd alors de l'énergie, car il excite le matériau. En réflexion, on peut décrire une telle expérience en partant de la figure E1 de l'encadré 1, et en interprétant les deux flèches, incidente et réfléchie, soit comme des rayons $\mathrm{X}$ soit comme des électrons. On mesure la différence en énergie des deux faisceaux, qui correspond à l'énergie des excitations induites dans le matériau, et on reporte l'intensité du faisceau réfléchi en fonction de la perte d'énergie. Ceci donne la "fonction de perte", ou le "facteur de structure dynamique " qui lui est proportionnel.

La figure 5 a montre le facteur de structure dynamique obtenu par diffusion inélastique des rayons $\mathrm{X}$ pour le sodium. Ce métal est presque homogène, et correspond donc bien à l'image de l'eau avec une onde bien définie. On voit en effet dans le spectre un pic unique vers $6 \mathrm{eV}$ : il correspond à l'excitation d'un plasmon. Le calcul (ligne rouge) reproduit bien le résultat expérimental (cercles bleus).

Le plus souvent, cependant, la structure atomique et la composition chimique des matériaux donnent lieu à des spectres plus complexes. La figure $5 b$ montre la fonction de perte calculée pour le graphite, semimétal composé de couches empilées de carbone : deux structures bien distinctes apparaissent, correspondant aux oscillations de différents groupes d'électrons. À basse énergie, ce sont les électrons $\pi$, qui forment les liaisons (assez faibles) entre plans de carbone. À plus haute énergie, s'ajoutent les électrons $\sigma$, qui sont responsables des fortes liaisons entre atomes de carbone 

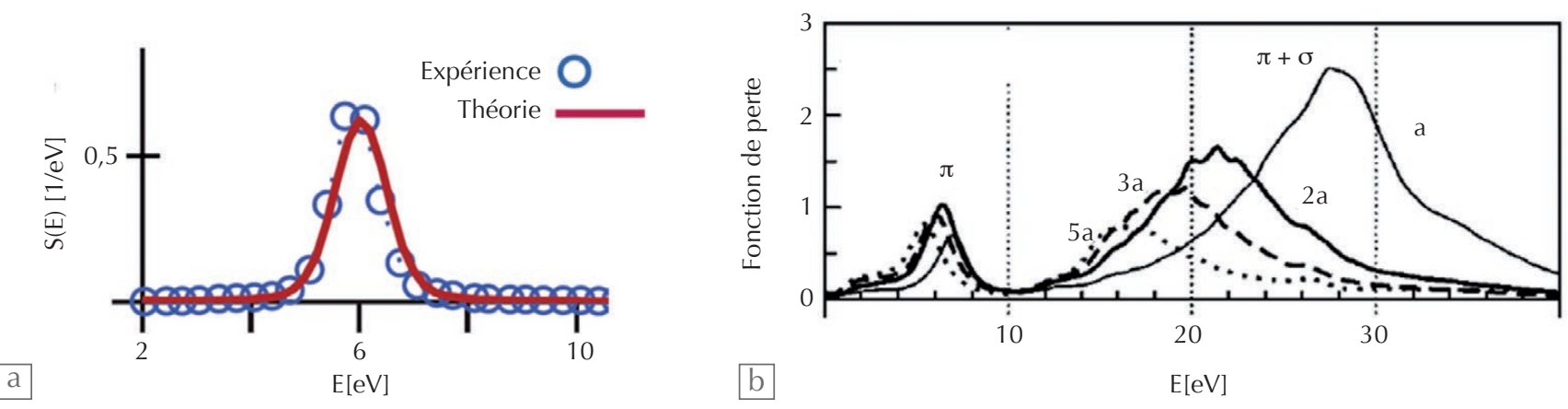

5. Spectres de plasmons. (a) Comparaison entre la théorie (courbe rouge) et le facteur de structure dynamique S(E) mesuré par diffusion inélastique des rayons X (cercles bleus) réalisée sur le sodium (M. Cazzaniga et al., Phys. Rev. B 84 (2011) 075109). (b) Comparaison entre les fonctions de perte théoriques obtenues pour différentes distances entre les plans de graphite, multiples de la distance d'équilibre a. On voit deux structures plasmoniques, la première ayant une position relativement stable, tandis que la seconde est très sensible à la distance entre plans. (A.G. Marinopoulos et al., Phys. Rev. B 69 (2004) 245419).

\>

dans un plan. Après avoir vérifié que le calcul est en bon accord avec des mesures, nous pouvons analyser différents effets. Par exemple, on peut tester l'importance de l'interaction entre plans de graphite, en étudiant comment le spectre change lorsqu'on éloigne les plans. Les calculs montrent que le plasmon constitué des électrons $\pi$ et $\sigma$ bouge beaucoup, tandis que le plasmon $\pi$ ne bouge presque pas (fig. 5b). On peut ainsi modifier aisément les paramètres dans le calcul pour comprendre l'influence de différents facteurs, contrairement aux expériences !

Revenons à la propagation d'une particule. Elle perturbe le système, ce qui peut exciter des plasmons, et donc donner lieu à des satellites (fig. 2). Ceci passe par la polarisation dynamique du système, créée par une superposition de nombreuses paires électron-trou. Cette information est contenue dans l'interaction écrantée, schématisée dans la figure $3 b$.

Pour en tenir compte, quand on veut décrire la propagation d'un électron, on remplace l'interaction nue dans le diagramme d'échange par l'interaction écrantée. Finalement, chaque processus peut apparaître plusieurs fois : la particule se propage, rencontre les autres, change sa propagation, fait une nouvelle rencontre, etc. L'histoire de la propagation, qui est la somme de toutes ces possibilités, donne alors lieu à une équation appelée « équation de Dyson ", qui se traduit en diagrammes comme celui de la figure $3 \mathrm{c}$.

Pour être précis, cette figure est encore loin de correspondre à l'équation de Dyson complète : elle représente l'approximation dite "GW » (du fait qu'elle se construit de façon simple avec la fonction de Green appelée $G$, et l'interaction écrantée appelée W) [4]. Cette approximation est très bonne en général pour calculer des propriétés liées aux quasi-particules, comme les bandes interdites dans les solides ; mais elle est plutôt mauvaise pour les satellites. Un de nos centres d'intérêt est d'améliorer la description des satellites ; nous développons donc des méthodes pour prendre en compte plus de processus (autrement dit, plus de diagrammes) qui sont importants dans ce contexte. La figure 6 montre un de nos travaux récents : il s'agit du spectre de photoémission du silicium en volume.

Les bandes de valence du silicium se situent entre 0 et $-12 \mathrm{eV}$. Cette partie du spectre correspond donc qualitativement à ce que l'on attendrait dans un schéma avec des électrons indépendants ; ce sont les quasi-particules. Tout le reste du spectre expérimental (croix bleues) est constitué par des satellites plasmoniques. Le calcul GW donne la courbe rouge, très satisfaisante pour les bandes de valence, mais non pas pour les satellites. Notamment, un seul satellite est présent, tandis que l'expérience en montre clairement au moins deux. Pour trouver cette création de plasmons multiples, il faut aller au-delà de l'approximation GW. Dans une première étape, nous avons adapté au contexte des calculs modernes une approche historique, dite "de développement en cumulants " (du fait qu'on peut l'obtenir à partir d'un développement d'une fonction exponentielle). Le résultat est la courbe verte. On voit apparaître la forme du spectre attendue. Finalement, si on inclut d'autres phénomènes tels que la perte d'énergie d'un électron sur son chemin pour arriver au détecteur, on obtient la courbe noire, en très bon accord avec les expériences.

Notons que ces satellites et autres excitations secondaires sont intéressants du point de vue fondamental, mais aussi technologique : on peut penser par exemple à la création de paires électron-trou multiples par un seul photon, un des espoirs pour le photovoltaïque.

\section{Pas si simple: ce qui reste à faire}

Est-ce tout ? Loin de là. Regardons de nouveau le bateau de la figure 4a. Nous considérons l'eau comme une matière continue et homogène, tout en sachant qu'elle est composée de molécules. Ceci ne nous semble pas important - par rapport à la taille du bateau, les molécules et la distance entre elles sont très petites. Mais pour l'électron qui se propage dans le système, une telle différence de taille n'existe pas, surtout si le système électronique n'est pas très dense. On voit alors apparaître des phénomènes qui vont au-delà de ce que l'on peut décrire avec des ondes classiques.

Pour comprendre ceci, regardons un système très simple : un dimère modèle (fig. 7a). Il s'agit d'un système composé de deux sites, chacun avec une orbitale qui peut contenir deux électrons. Ces orbitales s'hybrident et forment deux états, l'état liant de plus basse énergie, et l'état anti-liant de plus haute énergie. Supposons qu'il y ait déjà un électron dans le système - qui ressemble donc à la molécule $\mathrm{H}_{2}{ }^{+}-$, et qu'on y introduit un deuxième électron, de spin opposé. Que va-t-on observer 
dans une expérience de photoémission inverse ?

Si les deux sites sont proches, on va pouvoir représenter le premier électron, qui se trouve dans l'état liant, comme une densité classique distribuée sur les deux sites (fig. 7a). L'introduction du deuxième électron va donner lieu à ce que nous avons vu auparavant : un pic de quasi-particule et un satellite dû à l'excitation de la densité existante.

En revanche, si les sites sont éloignés, les énergies des états liant et antiliant se rapprochent, l'hybridation des orbitales sur les deux atomes disparaît, et le premier électron est soit à gauche, soit à droite, avec la même probabilité (fig. 7b). Le deuxième électron va donc, avec la même probabilité, arriver soit sur un site vide, soit sur un site déjà occupé où il sera soumis à une répulsion coulombienne. La fonction spectrale montre alors deux pics aux énergies bien distinctes (fig. 7c). Un tel effet n'est pas prévu par l'approximation GW, qui ne "voit " pas les électrons du système en détail et ne prévoit donc qu'un seul pic, au milieu, car elle interprète la densité - qui est toujours symétrique - comme une distribution de charge classique. Nous entrons ici dans le domaine des phénomènes que l'on peut appeler " de corrélation forte ", car il faut explicitement corréler le mouvement d'au moins deux particules.

Bien sûr, pour améliorer l'approximation $\mathrm{GW}$, on pourrait continuer à ajouter des diagrammes à la théorie, en rendant l'histoire toujours plus "vraie ", et les calculs toujours plus complexes... mais on comprend aisément qu'une telle procédure rencontre rapidement ses limites.

Actuellement, notre but est de mettre en place une approche alternative [5]. Nous partons pour ceci d'une équation différentielle fonctionnelle pour la fonction de Green en présence d'un potentiel externe dépendant du temps. Cette équation est connue depuis des décennies, mais elle a essentiellement servi à produire des séries limitées de diagrammes (l'approximation GW, par exemple). Notre idée est de tenter une solution directe de l'équation différentielle, bien sûr approchée, en évitant les hypothèses courantes (qui sont par exemple soit une interaction faible, soit une interaction locale). La figure 6 est en fait un premier résultat de cette stratégie. Pour aller au-delà, nous devrons abandonner le langage des diagrammes qui nous a accompagnés jusqu'ici. Mais ça, c'est une autre histoire...

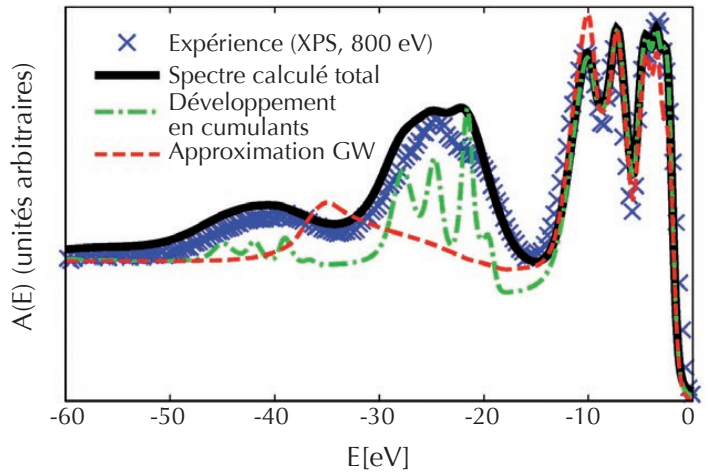

6. Spectre de photoémission du silicium en volume. Croix bleues : résultats expérimentaux. Rouge : calculs GW. Vert : fonction spectrale obtenue par des calculs utilisant des cumulants. Courbe noire : spectre total (voir texte) basé sur un développement en cumulants (M. Guzzo et al., Phys. Rev. Lett. 107 (2011) 166401).
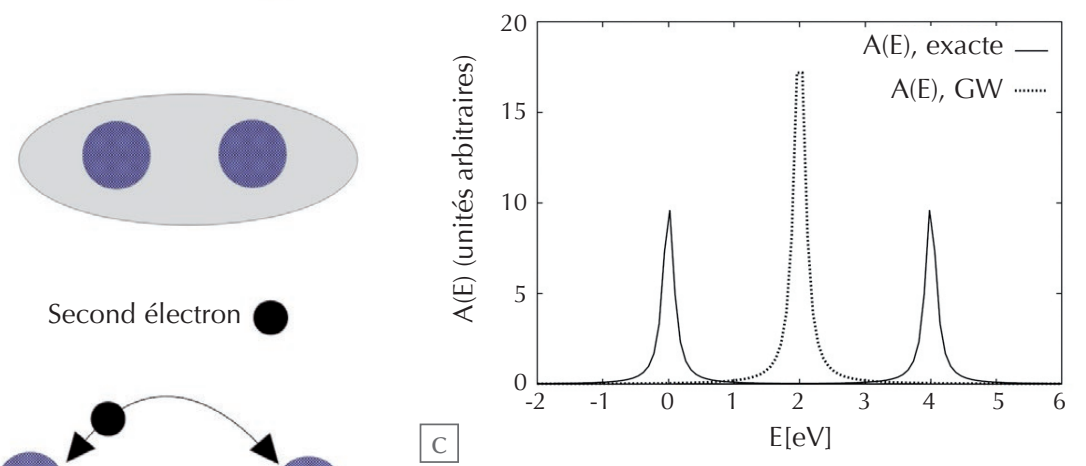

$\mathrm{b}$

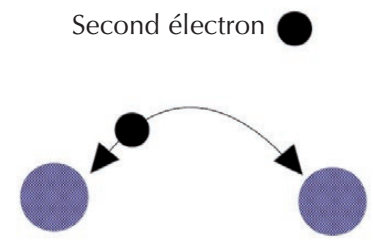

7. Excitations électroniques d'un dimère modèle, avec un électron qui occupe une liaison moléculaire, correspondant à l'ion $\mathrm{H}_{2}^{+}$.

(a) Quand les atomes sont proches, on peut penser que la charge de l'électron, indiquée par la région entourée par une ellipse, est distribuée de façon symétrique sur les deux atomes.

(b) Quand on dissocie la molécule, on réalise qu'on trouve l'électron soit à droite soit à gauche, comme le montre la flèche, avec la même probabilité : on ne peut plus interpréter la densité électronique comme une distribution de charge classique.

(c) Fonction spectrale calculée du dimère de la figure (b), correspondant à $\mathrm{H}_{2}^{+}$avec une grande distance entre les atomes. On ajoute un second électron, ce qui correspond à la photoémission inverse. Pour une grande distance entre atomes, il faut tenir compte du fait que l'électron du système se trouve soit à droite, soit à gauche. l'électron ajouté peut alors tomber soit sur un site vide (pic d'énergie zéro), soir sur un site déjà occupé par le premier électron (pic déplacé plus haut en énergie par l'interaction coulombienne). L'approximation GW ne peut pas reproduire cet effet : elle crée toujours un seul pic, au milieu (courbe en pointillés). (P. Romaniello et al., J. Chem. Phys. 131 (2009) 154111).

\section{Références}

1• P. Fulde, Correlated Electrons in Quantum Matter, World Scientific (2012).

2•W. Kohn, "Nobel Lecture: Electronic structure of matterwave functions and density functionals", Rev. Mod. Phys., 71 (1999) 1253

3 Parmi les bons livres sur ce sujet, voir par exemple, l'introduction très abordable dans R.D. Mattuck, $A$ Guide to Feynman Diagrams in the Many-Body Problem, Dover Books on Physics (1992).
4 L. Hedin, "New Method for Calculating the One-Particle Green's Function with Application to the Electron-Gas Problem", Phys. Rev. 139 (1965) A796-A823 ; "On correlation effects in electron spectroscopies and the GW approximation", J. Phys. C, 11 (1999) R489.

5• G. Lani, P. Romaniello et L. Reining, “Approximations for many-body Green's functions: insights from the fundamental equations", New J. Phys., 14 (2012) 013056. 